\title{
Vegetación arbórea de una sección del bosque secundario de la Estación Biológica Pindo Mirador
}

\author{
Tree vegetation of a section of the secondary forest of the Pindo Mirador \\ Biological Station
}

Christopher Oswaldo Paredes Ulloa. ${ }^{1}$, Jorge Ferro Díaz. ${ }^{2} \&$ Pablo Lozano Carpio. ${ }^{3}$

Recibido: 18-07-2020 / Revisado: 12-08-2020 / Aceptado: 14-09-2020 / Publicado: 03-10-2020

\begin{abstract}
.
DOI: https://doi.org/10.33262/cienciadigital.v4i4.1435

The secondary forest of the Biological Station Pindo Mirador, province of Pastaza, Ecuador, have been poorly studied, proposing with the research, to characterize the tree structure in a sector of the forest area of the Station. Above the sea level, four plots of 0.1 ha were established covering a sampling area of 0.4 ha; all trees with diameter a 1.30 from the soil $\geq 10 \mathrm{~cm}$ were measured, determining their absolute height. With the measurement records, four diametric classes and similar number of height ranges were established. Species and plot abundances, diametric classes and height ranges were analyzed to extract their descriptive statistics, and by nonparametric comparisons to establish differences between them. The contribution of species to the forest structure was assessed by determining structural diversity indexes, including the Importance Value Index and the basal area for each. The results show a richness of 49 species belonging to 36 genera of 27 families, where trees with diameters between 10 and $20 \mathrm{~cm}$ and heights between 8 and $20 \mathrm{~m}$ predominate. It is concluded that it is a moderately homogeneous forest with typical elements of the Andean-Amazon transition, of low species richness, whose vertical structure of the dominant flight is of the medium floor or subcanopy, medium dense, dominated by
\end{abstract}

${ }^{1}$ Universidad de Pinar del Río, Programa de Doctorado en Ciencias Forestales, Pinar del Río, Cuba, email chris9enginer@gmail.com

${ }^{2}$ Centro de Investigaciones y Servicios Ambientales ECOVIDA, Departamento de Investigaciones, Ministerio de Ciencia Tecnología y Medio Ambiente, Pinar del Río, Cuba. jorge.ferro2011@ gmail.com

${ }^{3}$ Universidad Estatal Amazónica, Centro de Investigación y Posgrado para la conservación Amazónica CIPCA, Herbario ECUAMZ, Puyo, Pastaza, Ecuador.pablo_lozano@yahoo.com 
trees of Hyeronimaoblonga and Piptocoma discolor, which have the biggest influence on the structure.

Keywords: Regeneration forests, dasometry, diversity indexes, species Importance Value Index.

\section{Resumen.}

El bosque secundario de la Estación Biológica Pindo Mirador, provincia de Pastaza, Ecuador, han sido pobremente estudiados, proponiéndose con la investigación, caracterizar su estructura arbórea. Se establecieron cuatro parcelas de 0.1 ha que cubren un área de muestreo de 0.4 ha; fueron medidos todos los árboles con diámetro a 1.30 del suelo $\geq 10 \mathrm{~cm}$, determinándose además su altura absoluta. Las abundancias por especies y parcelas, clases diamétricas y rangos de altura fueron analizadas para extraer sus estadísticos descriptivos, y mediante comparaciones no paramétricas establecer diferencias entre ellos. Se evaluó la contribución de especies a la estructura del bosque mediante la determinación de Índices de diversidad estructural, incluyendo el Índice de Valor de Importancia de especies, y su área basal. Los resultados muestran una riqueza de 49 especies pertenecientes a 36 géneros de 27 familias, donde predominan árboles con diámetros entre 10 y $20 \mathrm{~cm}$ y alturas entre 8 y $20 \mathrm{~m}$. Se concluye que es un bosque relativamente homogéneo, con elementos típicos de la transición andino-amazónica, de baja riqueza de especies, cuya estructura vertical del vuelo dominante es del piso medio o subdosel, medianamente denso, dominado por árboles de Hyeronima oblonga y Piptocoma discolor, las que tienen la mayor incidencia en su estructura.

Palabras claves: Bosques de regeneración, dasometría, índices de diversidad, Índice de Valor de Importancia de especies.

\section{Introducción.}

Los bosques se consideran un arma natural contra el calentamiento mundial debido a su capacidad para mitigar el cambio climático reduciendo las emisiones de carbono ocasionadas por la deforestación y la degradación forestal (FAO, 2018); estas afectaciones, han sido consideradas como las causas de mayor impacto en la conservación de biodiversidad en el Ecuador (León-Yánez et al., 2011).

Según reporta el Ministerio del Ambiente del Ecuador (2015), entre 2008 y 2012 la tasa de deforestación en el país fue de $(0,65 \%), 65880$ ha año ${ }^{-1}$; la fuente citada refiere que dicha tasa es inferior a la que se había determinado para el período 2000 a 2008 (75.300 ha año ${ }^{-1}$ $42 \%$ menos que en el período anterior), resultado de la consolidación de los derechos de propiedad de las áreas forestales remanentes más importantes. 
Una de las estrategias implementadas por Ecuador para conservar áreas forestales importantes ha sido la declaración de Bosques Protectores, categoría establecida en la Ley Forestal de 2001 (Aguirre, 2018).

El área forestal que ocupa hoy la Estación Biológica Pindo Mirador es propiedad del Gobierno Autónomo Descentralizado provincial de Pastaza, la cual se ha declarado con el propósito de conservar el ecosistema forestal que protege la captación de aguas de la cuenca del Río Pindo Grande, implementado varias acciones que favorecen la conservación, destacando el fomento de la investigación científica (Luzuriaga et al., 2011). Reconociendo la Estrategia para Implementar las Recomendaciones de la Propuesta de Pucallpa sobre el Desarrollo Sostenible del Bosque Secundario de la Región Amazónica (Toledo, 1999) para los cuales se requiere realizar investigaciones científicas básicas orientadas principalmente al conocimiento de la dinámica de los bosques y estadios sucesionales.

En la Estación Biológica Pindo Mirador se desarrollaron estudios hace ya más de 10 años, caracterizando sectores y componentes del ecosistema presente, y en particular sobre el bosque secundario fueron realizados inventarios y otras descripciones, principalmente en el sector de la Laguna Pindo, cubriendo un área reducida de muestreo (Luzuriaga, 2014).

Teniendo en cuenta los elementos expuestos, con la presente investigación se propuso como objetivo, caracterizar la estructura arbórea del bosque secundario de la Estación Biológica Pindo Mirador, aportando información actualizada que apoye las acciones de conservación que en ella se desarrollan.

\section{Metodologia.}

La Estación Biológica Pindo Mirador (EBPM) tiene un área de 248 ha, ubicadas en el cantón Mera de la Provincia Pastaza, Ecuador, entre los 1150 - 1300 m.s.n.m. La temperatura media varía entre $18{ }^{\circ} \mathrm{C}$ y $24{ }^{\circ} \mathrm{C}$, recibiendo lluvias que oscilan entre los 4000 y 5300 milímetros anuales, encontrándose en una de las áreas más lluviosas que se encuentran en Ecuador (Ministerio de Ambiente del Ecuador, 2013).

El territorio se ubica en el sector biogeográfico norte de la Cordillera Oriental de los Andes, donde se presenta el ecosistema de bosque siempreverde piemontano (Ministerio de Ambiente del Ecuador, 2013). El sector donde se aplicó el muestreo dentro de este estadio sucesional se ubica entre las alturas 1208 m.s.n.m. (coordenadas S1 ${ }^{\circ} 26^{\prime} 42.11301 "$ y W78²'31.76349") y 1292 m.s.n.m. (coordenadas S1²6'45.81462" y W78²'31.4906").

Unidad de muestra (n): 0.1 ha (20 X 50 m) siguiendo a González-Oliva et al. (2017).

Tamaño de muestra $(\mathrm{N}): 4$ parcelas de 0,1 ha $(0.4$ ha) que representa el 9\% del área total que ocupa este estadio sucesional. 
Diseño de muestreo: sistemático, con separación irregular entre parcelas, según las variaciones del terreno, entre 70 y $100 \mathrm{~m}$.

Mediciones de campo: La determinación de especies se hizo directamente en el campo; las que no fue posible determinar en la propia área, se le tomaron muestras o fotografías de las estructuras claves, trasladándose a condiciones de colección para ser contrastadas y precisar su determinación con el apoyo de literatura especializada, Catalogo de Plantas Vasculares del Ecuador (Jorgensen y León-Yánez, 1999). Libro rojo de las Plantas Endémicas del Ecuador (León-Yánez et al., 2011) y con el apoyo de expertos del Herbario ECUAMZ del CIPCA de la Universidad Estatal Amazónica.

Fueron medidos los diámetros del tronco de todos los individuos (incluyendo los muertos en pie, a los que se les registró con la categoría de Secos) que a la altura de $1.30 \mathrm{~m}$ del suelo (D1.30) tuvieran $10 \mathrm{~cm}$ o más, siguiendo a Lozano et al., (2013); para esto se utilizó una Forcípula Finlandesa FO102A. Considerando los criterios de Patiño et al., (2015) todo árbol cuya base se encontró sobre el borde de la parcela fue tomado en cuenta siempre que la mitad o más del área basal estuviera dentro de la misma. A cada individuo también se le midió su altura absoluta (h), utilizando un Clinómetro Suunto.

Se identificaron las especies tanto nativas como endémicas utilizando el Catálogo de Plantas Vasculares del Ecuador (Jorgensen; Leon-Yanez, 1999), y aquellas que poseen categoría de amenaza asignada, por la Lista Roja de la Flora Ecuatoriana (León-Yánez et al., 2011). Según la frecuencia observada de especies más los árboles muertos (Secos) por parcelas se concedieron categorías de Clases, y con ellas se confeccionó un histograma de presencia; las Clases establecidas fueron:

Clase IV: presentes en las cuatro parcelas

Clase III: presentes en tres parcelas

Clase II: presentes en dos parcelas

Clase I: presentes en una sola parcela (rara)

Al evaluar la estructura horizontal se determinaron los valores de abundancia, dominancia, y frecuencia relativa de cada especie y con ello se calculó el Índice de Valor de Importancia de Especies (IVIE) siguiendo a Curtis y Macintosh (1951). Como parte de la estructura horizontal se determinó la densidad (indiv. ha ${ }^{-1}$ ) y el área basal $(\mathrm{G})$ en $^{2}$ y en porcentaje, por especies, acorde a los criterios de Patiño et al., (2015) realizando además un análisis de la estructura por clases diamétricas, estableciéndose cuatro intervalos:

Clase diamétrica 1 (CD1): 10,0 a 19,9 cm

Clase diamétrica 2 (CD2): 20,0 a 29,9 cm 
Clase diamétrica 3 (CD3): 30,0 a 39,9 cm

Clase diamétrica $4(\mathrm{CD} 4): \geq 40,0 \mathrm{~cm}$

Los registros individuales de altura de cada árbol censado en las parcelas fueron agrupados en cuatro rangos, a saber:

Rango de altura 1 (RH1): 2,00 a 7,99 m

Rango de altura 2 (RH2): 8,00 a 13,99 m

Rango de altura 3 (RH3): 14,00 a 19,99 m

Rango de altura 4 (RH4): 20,00 a 25,99 m

Mediante el programa estadístico PAST versión 3.01 (Hammer et al., 2001) se realizó la determinación de índices de diversidad para el análisis estructural de la masa forestal, según criterios de Del Río et al., (2008) determinando la heterogeneidad mediante el índice de Shannon (H); la dominancia mediante el índice de Simpson (1-D) y la equitatividad mediante el Índice de uniformidad (Ev_e^H/S). También se estimó el número de especies que se esperarían en la comunidad arbórea mediante el índice de Chao2 (SChao2), para lo cual se siguen los criterios de Escalante (2003), permitiendo obtener un criterio valorativo de diversidad arbórea estimada respecto a la observada en el muestreo.

Dada la variabilidad observada de registros de abundancia de los arbóreos medidos en las parcelas, se valoraron sus estadísticos descriptivos y representación gráfica. Para evaluar si es posible detectar alguna diferenciación significativa o no de los registros de abundancia de arbóreos por cada una de las clases diamétricas establecidas, se aplicó una prueba no paramétrica de Kruskal-Wallis para la mediana por clases, cuyos resultados podrían apoyar el análisis de la estructura horizontal del bosque.

\section{Resultados.}

Composición de especies: fueron identificadas 49 especies de árboles pertenecientes a 36 géneros de 26 familias. Del total de géneros ocho agrupan el 40,8\% de la riqueza específica en el área, siendo Miconia el que reporta el mayor registro con seis especies; otros siete géneros (Cordia, Guatteria, Hyeronima, Palicourea, Saurauia, Viburnum y Vismia) presentan dos especies cada uno, y los restantes 28 solo con una. Las familias con mayor representatividad en el muestreo fueron Melastomataceae (siete especies), Rubiaceae, Myrtaceae, Euphorbiaceae y Fabaceae, con tres especies cada una.

Del total de especies dos son endémicas, Saurauia aequatoriensis Sprague y Nectandra coeloclada Rohwer, las que son reconocidas como especies amenazadas (León-Yanes et al., 2011). 
Los resultados obtenidos respecto a la composición de especies y familias de los arbóreos identificados, coinciden con los que reporta Luzuriaga (2014) en cuanto a las familias con mayor riqueza de especies, la que evaluó sectores del mismo ecosistema, sin embargo en su caso fue Rubiaceae la familia de más altos registros; también hay semejanza con lo que exponen Sánchez et al. (2017) quienes en dos sectores muy cercanos (Mera y Shell), evaluando bosques siempreverdes perturbados, detectaron coincidencias en la dominancia de Euphorbiaceae, Melastomataceae y Fabaceae principalmente; tales semejanzas refuerzan el criterio de los autores citados, acerca de que estas familias constituyen elementos distintivos del bosque húmedo tropical con influencia antrópica, caracterizados por elementos de regeneración pionera de rápido crecimiento.

El bajo porcentaje de endemismo que se observa en la flora puede estar asociado a dos factores fundamentales; uno relacionado por la previa desaparición de especies de tala selectiva, otro por la ubicación del ecosistema, típico de una transición entre la Amazonía baja y los sistemas de bosques andinos (Ministerio de Ambiente del Ecuador, 2013), al respecto, León-Yanes et al. (2011) reportan el endemismo de la Amazonía ecuatoriana alrededor del 10.7\%, sin embargo Jørgensen et al. (2011) refiere que en Ecuador, la manifestación del endemismo y cantidad de especies amenazadas, a las alturas entre cero y 1000 m.s.n.m. puede fluctuar entre 5 y $10 \%$ al analizarlos comparativamente con otros países andinos; esto confirma que porcentajes bajos de endemismo y especies amenazadas es una característica típica de la diversidad del bosque; el otro factor está relacionado con las intensas extracciones madereras a que fue sometido el territorio que ocupa hoy el bosque secundario (Luzuriaga et al., 2011).

Las mayores frecuencias, según el porcentaje de presencia de los arbóreos en las parcelas del muestreo (Figura 1), lo obtuvieron S. aequatoriensis, P. discolor, H. oblonga, B. pentamera y A. guianensis, las que estuvieron presentes en el $100 \%$ de las muestras y son las que representan la riqueza que abarca la Clase de Presencia IV; la Clase III también registró cinco especies (M. soegengii, M. quadripora, P. guianensis, T. occidentalis y C. angustifolia), ambas Clases contienen las especies más frecuentes en el área de estudio.

Figura 1. Histograma de presencia con la cantidad de especies por cada una de las Clases establecidas.

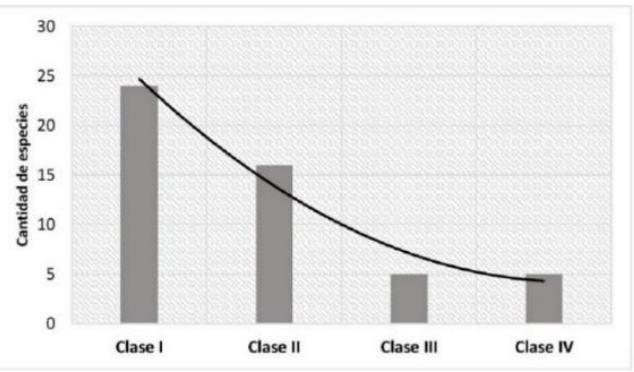

Fuente: Elaboración propia. 
El cálculo del Índice de Valor de Importancia (IVI) de las especies, 10 resultaron las que mayor valor porcentual obtienen (Tabla 2), siendo cuatro de ellas las que por su valor superior al $5 \%$, aportan más a la estructura de masa forestal (H. oblonga, P. discolor, $M$. calvescens y $M$. pilgeriana).

Tabla 2. Valor de importancia de las especies con mayor contribución a la estructura del bosque secundario en la Estación Biológica Pindo Mirador; se muestran los parámetros que integraron el análisis del Î́ndice de Valor de Importancia (IVI), a saber, Abundancia Relativa (AR), Frecuencia Relativa (FR) y Densidad Relativa (DR).

\begin{tabular}{lllll}
\hline \multicolumn{1}{c}{ Especies } & \multicolumn{1}{c}{ AR } & \multicolumn{1}{c}{ FR } & DR & \multicolumn{1}{c}{ IVI } \\
\hline Hyeronima oblonga & 13,92 & 4,44 & 4,45 & 7,60 \\
Piptocoma discolor & 6,23 & 3,33 & 11,85 & 7,14 \\
Miconia calvescens & 6,96 & 2,22 & 6,71 & 5,30 \\
Miconia pilgeriana & 7,33 & 2,22 & 6,14 & 5,23 \\
Cecropia angustifolia & 5,49 & 3,33 & 5,89 & 4,90 \\
Miconia quadripora & 6,23 & 3,33 & 4,86 & 4,81 \\
\hline
\end{tabular}

Abundancia: Fueron censados 339 individuos arbóreos en las cuatro parcelas del muestreo; esta abundancia real del componente arbóreo inventariado representa una densidad de 847 indiv. ha ${ }^{-1}$.

Fuente: Elaboración propia.

Las especies que mayor valor de IVI obtienen (Tabla 2) son las que más aportan a la conformación estructural de este ecosistema de bosque siempreverde piemontano en estadio secundario, tanto por la frecuencia de aparición como por la abundancia que observan, convirtiéndose en las dominantes del componente arbóreo. Este resultado coincide con el que obtuvo Luzuriaga (2014) en el mismo ecosistema, principalmente $P$. discolor, $M$. splendens y $C$. angustifolia. Coincidimos en que estas especies absorben gran cantidad de nutrientes lo que conlleva a que ocupen mayor espacio físico en el bosque y controlen un elevado porcentaje de energía que llega al sistema.

La variabilidad de registros de abundancia confirma su estadio sucesional con una dinámica típica de bosque secundario, acorde a como se han expuesto definiciones para este tipo de dinámica pos disturbios (Ferro-Diaz y Fernández, 2013; Toledo, 1999). Un análisis comparativo con un estudio anterior en dicha área no aporta información concluyente pues Luzuriaga (2014) y Luzuriaga et al. (2011) asumieron un mayor rango de variación de mediciones, partiendo del D1.30 de los individuos igual o superior a $2.5 \mathrm{~cm}$, no obstante, es posible confirmar una densidad superior a otros estudios en ecosistemas semejantes (Sánchez et al., 2017). 
El valor medio de la riqueza que se reporta en el presente estudio es de 24 especies, y los resultados del estimado que hace la medida de riqueza de especies Chao2, indican valor de probabilidad superior $(\mathrm{SChao}=61.16$; desv est $=6.381)$. Con respecto al valor que reporta el Índice de Chao2, con estimado casi tres veces superior al observado indica que es un ecosistema de riqueza medianamente baja, coincidiendo así con Luzuriaga (2014); estudios futuros deberán profundizar en este análisis a partir de una ampliación del tamaño de muestra.

Estructura por clases diamétricas: Acorde a los cuatro rangos establecidos para definir las Clases diamétricas, se observó que en el ecosistema abundan más los arbóreos cuyos diámetros de troncos están entre 10.0 y $20.0 \mathrm{~cm}$ de D1.30 (CD 1). En la (Figura 2), se muestra el comportamiento de la media de abundancia por Clases diamétricas, donde la cantidad de arbóreos de menor diámetro representa el 59\% de la abundancia total que existe en esta masa forestal. Son significativas las diferencias en la variabilidad observada de abundancia por clases según expone el estadístico de contraste de Kruskall-Wallis $(\chi 2=49.64 ; \mathrm{gl} 3 ; \mathrm{p}=0.000)$

Figura 2. Distribución de la media de abundancia de arbóreos por Clases diamétricas. CD1: 10.0 y $1 ., 9 \mathrm{~cm}, \mathrm{CD} 2$ : entre 20.0 y 29.9 cm, CD3: entre 30.0 y 39.9; $\mathrm{CD} 4 \geq 40.0 \mathrm{~cm}$.

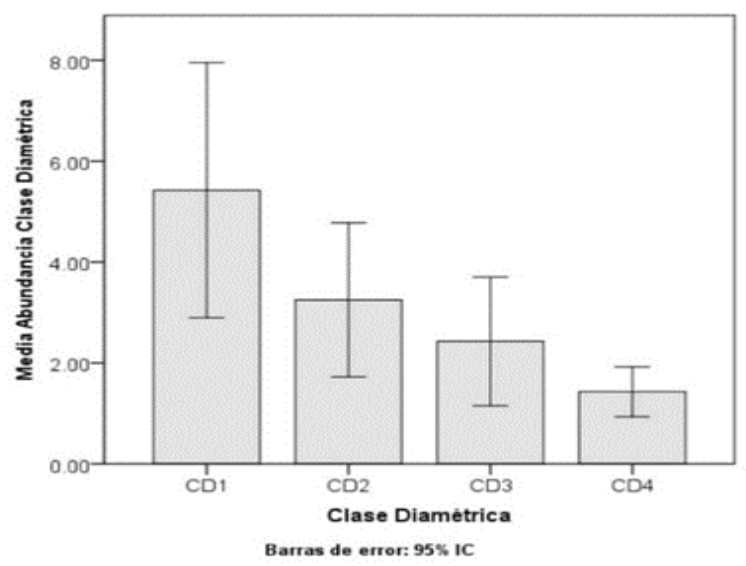

Fuente: Elaboración propia.

Área basal: El área basal determinada resalta la importancia de tres especies con valores superiores a $1 \mathrm{~m}^{2} P$. discolor $2.23 \mathrm{~m}^{2} ;$ P. guianensis $1.36 \mathrm{~m}^{2}$ y M. splendens $1.20 \mathrm{~m}^{2}$; otras cinco especies $C$. angustifolia $0.72 \mathrm{~m}^{2} ; H$. oblonga con valor de $0.71 \mathrm{~m}^{2} ; M$. quadripora 0.66 $\mathrm{m}^{2} ; V$. baccifera $0.44 \mathrm{~m}^{2}$ y $B$. pentámera $0.36 \mathrm{~m}^{2}$ sumadas a las tres antes mencionadas, representan el $65.32 \%$ del área basal total. Los análisis de esta variable dasométrica aportan información relevante para entender cómo se ajusta el ensamblaje de arbóreos en la estructura horizontal del bosque, principalmente para profundizar en el análisis de la densidad presente. 
Las especies que mayor presencia poseen en el inventario, agrupadas en las Clases IV y III (Figura 2), incluso varias de las que se listan en las otras dos Clases, han sido identificadas por otros autores dentro de la composición de especies del bosque siempreverde piemontano primario, tanto en la misma Estación Biológica Pindo Mirador (Luzuriaga, 2014) y como en áreas relativamente próximas de la provincia de Napo (Patiño et al., 2015; Torres et al., 2019); esto confirma que la matriz próxima de boques primarios, cuando el ecosistema se protege para su mantenimiento, contribuye a su dinámica sucesional progresiva. De igual forma, los árboles muertos, identificados como secos, tuvieron una frecuencia alta, con presencia en todas las muestras, elemento de importancia en el aporte de nutrientes al sistema forestal, que contribuye favorablemente a la mencionada dinámica sucesional (Del Río et al., 2008)

\section{Conclusión.}

- La Estación Biológica Pindo Mirador posee pocas especies que dominan por sus altas abundancias, medianamente denso, cuyos arbóreos predominantes son de troncos de diámetros inferiores a $20 \mathrm{~cm}$, y con estructura vertical del vuelo dominante es del piso medio o subdosel.

- En la composición de especies del ecosistema predominan arbóreos de familias ampliamente representadas en la Amazonía ecuatoriana, siendo más ricos en especies de Melastomataceae (21,36 \%), Euphorbiaceae (10,16\%), Rubiaceae (10,04\%) y Myrtaceae $(7,03 \%)$ destacando por su alta dominancia, frecuencia y abundancia Hyeronima oblonga, Piptocoma discolo, Miconia calvescens y Miconia pilgeriana como las especies con la mayor contribución a la estructura de la masa forestal.

- El bosque secundario evaluado tiene una complejidad estructural marcada por la dinámica pos disturbio, con elementos arbóreos típicos de la transición andinoamazónica, donde la complejidad está influenciada por la proporcionalidad de abundancias de especies.

\section{Referencias bibliográficas.}

Aguirre, Z., (2018). Biodiversidad ecuatoriana: estrategias, herramientas e instrumentos para su manejo y conservación. Universidad Nacional de Loja. Primera Edición. Loja, Ecuador. $136 \mathrm{p}$.

Curtis, J. T. and Mcintosh, R. P. (1951). An upland forest continuum in the prairie-forest border region of Wisconsin. Ecology 32(3): 476-496. http://dx.doi.org/10.2307/1931725 
Del Río, M., Montero, G., Montes, F., Cañellas, I. (2008). Revisión: Índices de diversidad estructural en masas forestales. Forest Systems 12(1): 159-176. https://recyt.fecyt.es/index.php/IA/article/view/2490

Escalante, T. (2003). ¿Cuántas especies hay? Los estimadores no paramétricos de Chao. Elementos 52: 53-56. https://www.redalyc.org/pdf/294/29405209.pdf

FAO. (2018). El estado de los bosques del mundo - Las vías forestales hacia el desarrollo sostenible. Roma, Italia.

Ferro-Diaz, J. and Fernández, J. (2013). Post disturbance dynamic of canopy gaps in semideciduous tropical dry forest from Guanahacabibes peninsula, Cuba; its relation with the abundance of vascular epiphytes. p. 200-213.

González-Oliva, L., Ferro-Díaz, J., Rodríguez-Cala, D., Berazaín, R. (2017). Métodos de inventario, monitoreo y colecciones biológicas. AMA ed. La Habana, Cuba. 502 pp.

Hammer, Ø.; Harper, D. A.; Ryan, P. D. (2001). Past: Paleontological statistics software package for education and data analysis. Palaeontologia electronica 4(1): 9. http://palaeo-electronica.org/2001_1/past/issue1_01.htm

Jørgensen, P. M., Ulloa, C., León, L., León-Yánez, S., Beck, S. G., Nee, M., Zarucchi, J. L.; Celis, M., Bernal, R., Gradstein, R. (2011). Regional patterns of vascular plant diversity and endemism. In: S.K. Herzog, R. Martínez, P.M. Jørgensen \& H. Tiessen (Editors). Climate Change and Biodiversity in the Tropical Andes. Inter-American Institute for Global Change Research (IAI) and Scientific Committee on Problems of the Environment (SCOPE), pp. 192-203. ciudad, estado y país de edición DOI: 10.13140/2.1.3718.4969

Jorgensen, P. M. y León-Yánez, S. (1999). Catálogo de las plantas vasculares del Ecuador. Missouri Botanical Garden. v. 75 faltan páginas, ciudad, estado y país de edición

León-Yánez, S.; Valencia, R.; Navarrete, H.; Pitman, N.; Endara, L.; Ulloa, C. (2011). Libro Rojo de Las Plantas Endémicas Del Ecuador, Segunda Edición. Herbario QCA, Pontificia Universidad Católica del Ecuador, Quito, Ecuador. 957 p.

Lozano, P., Torres, B., Rodríguez, X. (2013). Investigación de Ecología Vegetal en Ecuador: Muestreo y Herramientas Geográficas. Universidad Estatal Amazónica. Puyo, Ecuador. 158 p.

Luzuriaga, C., Cuasapaz, C., Quichimbo, G. (2011). Inventario forestal en la estación Pindo mirador. Tsafiqui-Revista Científica en Ciencias Sociales, n. 2: 83-107. https://doi.org/10.29019/tsafiqui.v0i2.211 
Luzuriaga, C. X. (2014). Inventario Florístico Del Bosque Que Rodea A La Laguna En La Estación Biológica Pindo Mirador Pastaza-Ecuador. Tsafiqui-Revista Científica en Ciencias Sociales 6: 15-25. https://doi.org/10.29019/tsafiqui.v0i6.228

Ministerio de Ambiente del Ecuador. (2013). Sistema de Clasificación de los Ecosistemas del Ecuador Continental. Subsecretaría de Patrimonio Natural Ed. Ecuador. http://app.sni.gob.ec/sni-

link/sni/PDOT/NIVEL\%20NACIONAL/MAE/ECOSISTEMAS/DOCUMENTOS/

Sistema.pdf

Patiño, J., Lozano, P., Tipán, C., Navarrete, H., López, R., Asanza, M., Torres, B. (2015). Composición florística y estructura de un bosque siempreverde piemontano de 600 a 700 m snm en la cuenca del río Piatúa, Napo, Ecuador. Revista Amazónica Ciencia y Tecnología 4(2): $166-214$ https://revistas.proeditio.com/REVISTAMAZONICA/article/view/218

Toledo, E. (1999). Estrategia para implementar las recomendaciones de la propuesta de pucallpa sobre el desarrollo sostenible del bosque secundario en región Amazónica. Tratado de Cooperación Amazonica, Secretaría Pro Tempore.

Torres, B., Vasseur, L., López, R., Lozano, P., García, Y., Arteaga, Y., Bravo, C., Barba, C., García, C. (2019). Structure and above ground biomass along an elevation small-scale gradient: case study in an Evergreen Andean Amazon forest, Ecuador. Agroforestry Systems, p. 1-11.

Sánchez, H. G. S., García, Y., Geada, G., Arteaga, Y., Rios, R., Guerero, J. (2017). Efecto del grado de antropización en la estructura, en tres sitios fragmentados bosque siempreverde piemontano. Revista Cubana de Ciencias Forestales 5(2):172-180.

\section{Ligital}




\section{PARA CITAR EL ARTÍCULO INDEXADO.}

Paredes Ulloa, C. O., Ferro Díaz, J., \& Lozano Carpio, P. (2020). Vegetación arbórea de una sección del bosque secundario de la Estación Biológica Pindo Mirador. Ciencia Digital, 4(4), 116-127. https://doi.org/10.33262/cienciadigital.v4i4.1435

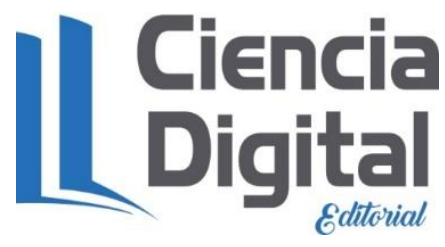

El artículo que se publica es de exclusiva responsabilidad de los autores y no necesariamente reflejan el pensamiento de la Revista Ciencia Digital.

El artículo queda en propiedad de la revista y, por tanto, su publicación parcial y/o total en otro medio tiene que ser autorizado por el director de la Revista Ciencia Digital.
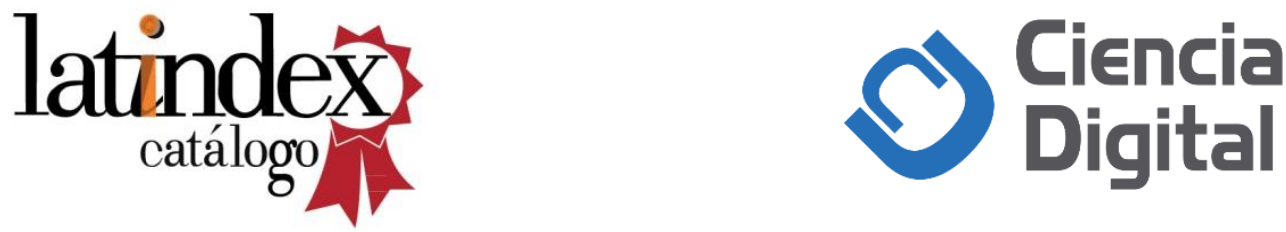